\title{
Smoking and health: a physician's responsibility. A statement of the joint committee on smoking and health
}

\author{
American College of Chest Physicians, American Thoracic Society, Asia Pacific Society \\ of Respirology, Canadian Thoracic Society, European Respiratory Society, International
}

Tobacco use, particularly cigarette smoking, is widely recognized by the medical community and the general public as a major public health problem. Physicians and medical organizations share a public health duty to address this problem. Physicians and their professional organizations must contribute effectively to measures undertaken to deal with cigarette smoking. The issues involved are complex and affect medical practice in a number of ways. The following statement, developed by six international organizations - the American College of Chest Physicians, the American Thoracic Society, the European Respiratory Society, the Asia Pacific Society of Respirology, the Canadian Thoracic Society, and the International Union Against Tuberculosis and Lung Disease - is intended to state the physicians responsibilities both to patients and to the community with regard to these general issues.

\section{Smoking and public health}

\section{The smoker}

Tobacco use is the single most important preventable risk to human health in developed countries, and an important cause of premature death worldwide. In countries which report death attributable to smoking (representing about one third of the world's population), annual deaths from smoking numbered about 1.7 million in 1985, with an estimated 2.1 million in 1995 (and hence about 21 million in the decade 1990-1999: 5-6 million in the European Community, 5-6 million in the USA, 5 million in former USSR, 3 million in Eastern Europe, and 2 million elsewhere). More than half of these deaths occur in people aged 35-69 yrs. During the 1990s, tobacco will cause about $30 \%$ of all deaths in people aged 35-69 yrs in developed countries (making it the largest single cause of premature death), plus about $15 \%$ of all deaths at older ages [1]. In addition, increasing incidence of smoking in the developing world is likely to lead to a new epidemic of smoking-related disease.

Smoking contributes to the onset of many diseases, and is thought to account for $87 \%$ of deaths from lung cancer, $82 \%$ from chronic obstructive pulmonary disease (COPD), 21\% from coronary heart disease (CHD), and $18 \%$ from stroke cases [2]. Therefore, once addicted to nicotine, the smoker faces an unacceptably increased risk of respiratory, neoplastic and cardiovascular disorders. Even without overt pulmonary symptoms, the smoker has a chronic inflammatory disease of the lower airways, with an accelerated decline in lung function.

\section{The nonsmoker}

Risk from tobacco smoke is not limited to the smoker. It has been estimated that exposure to environmental tobacco smoke (ETS) increases the risk of lung cancer by about $30 \%$ (about 3,000 cases a year in the USA) [3]. Nonsmoking infants and children who are chronically exposed to in utero and environmental tobacco smoke have an increased risk of respiratory disease, malignancy, and other health problems that result in increased hospitalization and days lost from school [4]. Nonsmoking adults who are exposed also have more respiratory symptoms that are likely to contribute to work absenteeism due to illness.

\section{Smoking cessation}

Smoking cessation has immediate and substantial health benefits, both symptomatically and pathophysiologically, and dramatically reduces the risk of most smokingrelated diseases [2]. One year after quitting, the risk of CHD decreases by $50 \%$, and within 15 yrs the relative risk of dying from CHD for an ex-smoker approaches that of a lifetime nonsmoker [5]. The relative risks of developing lung cancer, COPD and stroke also decrease, but more

Joint committee on smoking and health: American College of Chest Physicians, American Thoracic Society, Asia Pacific Society of Respirology, Canadian Thoracic Society, European Respiratory Society, International Union Against Tuberculosis and Lung Disease. S.I. Rennard, Chair of ACCP Delegation (University of Nebraska Medical Center, Omaha, NE); S. Weiss, Chair of ATS Delegation (Brigham and Women's Hospital, Channing Laboratory, Boston, MA); A.J. Woolcock, Chair of APSR Delegation (University of Sydney, Sydney, Australia); M. Lertzman, Chair of CTS Delegation (Winnipeg, MB, Canada); P. Paoletti, Chair of ERS Delegation (CNR Institute of Clinical Physiology, Pisa, Italy); N.E. Billo, Chair of IUATLD Delegation (IUATLD, Paris, France); C. Donner (Veruno Medical Center, Veruno, Italy); J.E. Henningfield (National Institute on Drug Abuse, Baltimore, MD); J. Moxham (King's College School of Medicine \& Dentistry, London, UK); N. Rigotti (Massachusetts General Hospital, Boston, MA); J. Samet (The Johns Hopkins University, Baltimore, MD); J. Snell (The Vanderbilt Clinic, Nashville, TN); A. Soffer (Master Fellow, ACCP, Northbrook, IL); J.L. Breeling (ACCP, Northbrook, IL). 
slowly. Ten to $14 \mathrm{yrs}$ after smoking cessation, the risk of mortality from cancer decreases to nearly that of people who have never smoked [6]. Smoking cessation shows a beneficial effect on pulmonary function, particularly in younger subjects, and the rate of decline among former smokers returns to that of lifetime nonsmokers [7]. Recent evidence shows that ceasing before the age of $35 \mathrm{yrs}$ is of greater benefit than ceasing at a more advanced age.

\section{Nicotine addiction}

The nicotine in tobacco products is highly addictive $[9,10]$. A greater percentage of casual users graduate to addictive patterns of use than occurs with cocaine, morphine or alcohol-containing substances [10,11]. Regular use of tobacco products is commonly associated with difficulty in achieving and sustaining abstinence, even when advised strongly by health professionals. Nicotine is the addictive agent in tobacco products, and is present in sufficient quantities in all commercially available tobacco products to cause and sustain addiction in children and adults [12]. All tobacco products are addictive; however, cigarettes appear to maximize the addictive potential of nicotine by requiring the user to inhale smoke into the lungs, thereby, resulting in extremely concentrated doses of nicotine being rapidly transmitted to the brain $[10,13]$.

\section{Physician-patient relationship}

Each physician is expected by the public, the medical profession and by each of his or her patients to prevent disease when possible, and to give the best available treatment once disease is present. This imposes upon all physicians the duty to ask each of their patients whether they smoke, and to provide proper information and counselling based on that history. Patients who are nonsmokers should receive positive reinforcement for decreasing the risk of smoking-related disease. A smoking patient requires a more detailed history of why and how much he or she smokes, whether there have been efforts to quit, any respiratory symptoms or disease from smoking, and a search for other risk factors which might increase the chance for that patient to develop cardiovascular disease, obstructive lung disease or lung cancer. Frank discussion of personal health risks, the benefits of stopping smoking, and methods available to assist them in stopping smoking are mandatory elements of high-quality care for every patient.

Paediatricians, obstetricians and family practitioners have a special opportunity to influence the health both of young patients and children. Education of pregnant women regarding harmful effects of smoking on themselves and their foetuses, and the risk of lower respiratory tract illness and symptoms in children growing up with smoking parents may help motivate the women to stop smoking before becoming severely addicted. Paediatricians and family practitioners should initiate counselling of children regarding harmful effects of smoking when the children are old enough to understand.

\section{Physicians and smoking cessation}

Physicians should explain to every smoking patient the medical risks associated with smoking and the reduction in risk associated with smoking cessation. Physicians should encourage abstinence, and prescribe follow-up on the use of specific smoking cessation programmes and strategies, such as self-help, behavioural or pharmacological approaches. A variety of behavioural programmes have been developed, and the physician should be able effectively to utilize resources available locally.

Pharmacological approaches to smoking cessation are currently based on nicotine replacement, and the physician should also be cognizant of these approaches. Nicotine replacement during early abstinence helps to relieve symptoms of withdrawal and can increase quit rates [14]. Nicotine delivered as a medication may also be addictive. However, the addictive potential of medications currently available appears to be related to their nicotine dosing characteristics. Thus, nicotine-delivering transdermal and Polacrilex gum medications appear to be of minimal addictive potential. Other symptoms under development, such as nasal sprays and vapour inhalers, may be of greater addictive potential but would still be expected to be lower in addictiveness and toxicity than tobacco products, which appear to optimize the addictive effects of nicotine due to their dosing and sensory characteristics.

\section{Physician as a role model}

Current and future physicians should be "exemplars" to their patients and communities. The physician should act as a role model by not smoking and by creating a smokefree environment in his/her office. Despite evidence of the negative health consequences, cigarette smoking is still highly prevalent among physicians in some countries [15]. Whilst smoking rates among physicians often reflect general population smoking rates, in most countries doctors smoke much less than the general population. Reduction of physician smoking is important, as the tutors of the people in matters of health have a responsibility to present a proper image. No suggestion should ever be made, particularly by physician behaviour, that smoking is not dangerous; therefore, physicians should not smoke in front of patients. Medical organizations should adopt active policies to establish physicians as role models with regard to smoking and health. Smoking prohibition in hospitals and in all structures associated with health-care should be mandatory, and such policies should be strongly supported by medical associations.

\section{Medical education}

Students in medicine and other professionals (technicians, nurses, etc.) must be taught from the first years of study about the negative effects of smoking, the addictive properties of nicotine, and how to help their future patients avoid smoking, if possible, and to quit smoking if needed. 


\section{Smoking prevention}

There is near universal agreement that those who start to smoke during teenage years are the most likely candidates for eventual nicotine dependence. Disease prevention, thus, begins by educating the young people. Obviously, therefore, cigarette advertising should not be directed to these younger age groups.

Unfortunately (and despite their pious disclaimers) the tobacco industry persists in preparing shrewd strategies to "entrap" the young smoker. Cartoon characters vie for the youngster's interest along with alternate techniques of publishing pictures of young vigorous, handsome, athletic men and women who find a particular brand of cigarette the most refreshing. This insidious indoctrination is worldwide and should be opposed by individual physicians and by medical organizations.

\section{Social action}

Many communities have recognized the social implications of smoking and have, therefore, enacted public policy and legislation. The goals of these policies are: 1) to prevent the initiation of smoking and the development of nicotine addiction; 2) to encourage the cessation of tobacco use among those who already smoke cigarettes or use other tobacco products; 3) to protect nonsmokers. Such policy includes: 1) taxes on cigarettes; 2) restrictions on advertising; 3 ) restriction on cigarette sales to children and teenagers; 4) prohibition of smoking in specified public places; 5) assurances that smokefree environments will be available in workplaces; 6) regulation of content and packaging of tobacco products; 7) public education; 8) promotion of smoking cessation services; 9) assistance for tobacco farmers; 10) restriction of international trade in tobacco; 11) health warnings on cigarette packages; and 12) abolition of "kiddie" packages of cigarettes. Such legislative issues are complex and involve balancing the rights and privileges of various heterogenous groups. Physicians have a special role in these considerations. In addition to their role as citizens, physicians are leaders with regard to any issue affecting public health. Physicians should, therefore, be aware that public policy regarding smoking can be an effective instrument of public health, and they have a responsibility to participate effectively in public debate, both as individuals and as members of medical organizations.

\section{Increase tobacco excise taxes}

Taxation of cigarettes is a special form of legislative remedy, and one of the most effective public policy approaches to smoking. Increases in the price of tobacco products have been demonstrated to reduce tobacco consumption among existing smokers and decrease the proportion of young people who begin to smoke. Taxation designed to decrease consumption should be strongly supported. Usually, this will involve using the tax system to ensure that tobacco prices are both high and steadily rising at a rate faster than inflation of prices and earnings. Studies suggest that price increases of a given percentage above inflation result in falls of consumption of about half that percentage. A $10 \%$ real price increase will typically result in a reduction in consumption of about $5 \%$.

Most governments use the funds raised by tobacco excise for general revenue. Governments should not fund routine services at the expense of public health. Alternative uses for such funds could include specific tobacco prevention and education programmes, including mass media campaigns, smoking cessation efforts among the poor, and helping tobacco farmers convert to other crops.

Such fiscal policies raise a number of important questions. A high price policy raises the possibility of smuggling and institution of appropriate control measures may be needed. A high price policy has also been criticized as an unfair penalty upon the poor. It is the physician's role to recognize and proselytize any perceived penalty in terms of health risks associated with cigarette smoking.

\section{Prohibit all forms of tobacco advertising and promotion}

The weight of evidence indicates that tobacco advertising and promotion increases tobacco consumption and encourages children to start to smoke. All forms of tobacco advertising and promotion should be prohibited. This should include such promotional efforts as the tobacco industry sponsorship of sporting events, where the products or company logo is displayed and there is a distribution of free cigarettes or coupon offers.

\section{Stop children's access to tobacco products}

Almost $90 \%$ of smokers start to smoke before $18 \mathrm{yrs}$ of age. Hence, young people are becoming addicted to nicotine before they may have acquired judgement to make a fully informed choice about tobacco use. Laws that ban the sale of tobacco products to minors should require the annual licensing of tobacco vendors and make licenses contingent on demonstrated compliance with the law. Because vending machines are also a source of tobacco products for children, the sale of cigarettes in vending machines should be banned.

\section{Prohibit smoking in public places and workplaces}

To protect nonsmokers from the documented health hazards of involuntary tobacco smoke, smoking should be banned in all public places, including all forms of public transport, sports arenas and other public facilities, all workplaces and restaurants.

\section{Regulate the content and packaging of tobacco products}

Tobacco products contain nicotine, which has been demonstrated to be a highly addictive drug. Therefore, the content and packaging of tobacco products should be subject to regulation by appropriate agencies. Tobacco product packages should carry adequate health information about the hazards of tobacco use and the addictive potential of nicotine. Existing warning labels on tobacco products and advertising should be expanded to include a specific warning about addiction to nicotine. 
Increase the amount and effectiveness of public education about tobacco

Because of the amount and pervasiveness of tobacco advertising, the general population is exposed to powerful and misleading prosmoking messages. The visibility of public education about tobacco, the addictiveness of nicotine, and the importance and methods of smoking cessation, should be increased.

Increase the availability and coverage of smoking cessation services

Although smoking cessation clearly benefits health, smoking cessation services (including behavioural counselling and nicotine replacement therapy) are inadequately covered by many existing health insurers. Effective smoking cessation techniques, including behaviour counselling and medication, should be covered both by public and private health insurers.

\section{Assist tobacco farmers in switching to other crops}

Governments should reduce support for tobacco farming and offer incentives to tobacco farmers to switch to other crops.

\section{Regulate international trade in tobacco products}

International trade policy should not be used to promote tobacco sales.

\section{Summary}

Tobacco use, particularly cigarette smoking, is a major cause of preventable disease and premature death worldwide. Both smokers and nonsmokers exposed to environmental tobacco smoke are at risk. Cessation of smoking reduces risks. Although the addictive properties of nicotine can make cessation difficult, both medical interventions aimed at helping smokers quit and social policies aimed at control of cigarette smoking can have significant benefits. Physicians should play an active role in control of smoking by ensuring that counselling and pharmacological therapy are available for the individual smoker. Physicians should also participate in the public debate regarding smoking both individually and through medical organisations. As smoking represents a threat to the public health, physicians must take a strong and active role in seeking its control.

\section{References}

1. Peto R, Lopez AD, Boreham J, Thun M, C.H. Mortality from tobacco in developed countries: indirect estimation from national vital statistics. Lancet 1992; 339: $1268-1278$.

2. Office of the US Surgeon General. Reducing the health consequences of smoking: 25 years of progress. 1989.

3. Environmental Protection Agency. Respiratory health effects of passive smoking: lung cancer and other disorders. 1992.

4. White JR, Froeb HF, Kulik JA. Respiratory illness in nonsmokers chronically exposed to tobacco smoke in the workplace. Chest 1991; 100: 39-43.

5. US Department of Health and Human Services. Health benefits of smoking cessation. A report of the surgeon general, Washington, DC, DHHS publication No. (CDC) 90-8416, 1990.

6. Kawachi I, Colditz GA, Stampfer MJ. Smoking cessation in relation to total mortality rates in women. Ann Intern Med 1993; 119: 992-1000.

7. Ockene JK, Kuller LH, Svedsen KH, Meilahn E. The relationship of smoking cessation to coronary heart disease and lung cancer in the Multiple Risk Factor Intervention Trial (MRFIT). Am J Public Health 1990; 80: 954958.

8. Sherrill DL, Holberg CJ, Emright PL, Lebowitz MD, Burrows B. Longitudinal analysis of the effects of smoking onset and cessation on pulmonary function. $A m \mathrm{~J}$ Respir Crit Care Med 1994; 149: 591-597.

9. Centers for Disease Control. The health consequences of smoking: nicotine addiction. A report of the Surgeon General. 1988.

10. Centers for Disease Control and Prevention, Office of Smoking and Health. Preventing tobacco use among young people. DHHS Publication No. 017-001-004910, 1994.

11. Henningfield JE, Clayton R, Pollin W. Involvement of tobacco in alcoholism and illicit drug use. Br J Addiction 1990; 85: 279-292.

12. Food and Drug Administration, Drug Advisory Committee. Issues concerning nicotine-containing cigarettes and other tobacco products. Meeting 27; August 2, 1994.

13. Henningfield JE, Stapleton JM, Benowitz NL, et al. Higher levels of nicotine in arterial than in venous blood after cigarette smoking. Drug Alcohol Depend 1993; 33: 23-29.

14. Fiore MC, Jorenby DE, Baker TB. Tobacco dependence and the nicotine patch: clinical guidelines for effective use. J Am Med Assoc 1992; 268: 2687-2694.

15. Masironi R. The smoking habits of European doctor; new insights. Future Directions in Nicotine Replacement Therapy. Paris, 1993. 